ESAIM: PROCEEDINGS AND SURVEYS, May 2021, Vol. 70, p. 1-13

Arnaud Duran, Benoît Fabrèges, Pauline Lafitte, Frédéric Lagoutière, Fabien Marche, Frédéric Rousset

\title{
NUMERICAL INVESTIGATIONS OF THE COMPRESSIBLE NAVIER-STOKES SYSTEM
}

\author{
Bilal Al-Taki ${ }^{1,2}$, Kevin Atsou ${ }^{3,4}$, Jean-Jérôme Casanova ${ }^{5,6}$, Thierry Goudon ${ }^{3,7}$, \\ Pauline Lafitte $^{8,9}$, Frédéric Lagoutière ${ }^{10,11}$ and Sebastian Minjeaud ${ }^{3,12}$
}

\begin{abstract}
In this paper we write, analyze and experimentally compare three different numerical schemes dedicated to the one dimensional barotropic Navier-Stokes equations:

- a staggered scheme based on the Rusanov one for the inviscid (Euler) system,

- a staggered pseudo-Lagrangian scheme in which the mesh "follows" the fluid,

- the Eulerian projection (on a fixed mesh) of the preceding scheme.

All these schemes only involve the resolution of linear systems (all the nonlinear terms are solved in an explicit way). We propose numerical illustrations of their behaviors on particular solutions in which the density has discontinuities (hereafter called Hoff solutions). We show that the three schemes seem to converge to the same solutions, and we compare the evolution of the amplitude of the discontinuity of the numerical solution (with the pseudo-Lagrangian scheme) with the one predicted by Hoff and observe a good agreement.
\end{abstract}

\section{INTRODUCTION}

We are interested in the simulation of the following Compressible Navier-Stokes system

$$
\begin{gathered}
\partial_{t} \rho+\partial_{x}(\rho u)=0, \\
\partial_{t}(\rho u)+\partial_{x}\left(\rho u^{2}\right)+\partial_{x} p-\partial_{x}\left(\mu \partial_{x} u\right)=0 .
\end{gathered}
$$

In (1), the unknowns $(t, x) \mapsto \rho(t, x)$ and $(t, x) \mapsto u(t, x)$ stand respectively for the density and the velocity of a fluid. The quantity $\mu>0$ is the viscosity of the fluid. We restrict to the isentropic case, where the pressure is

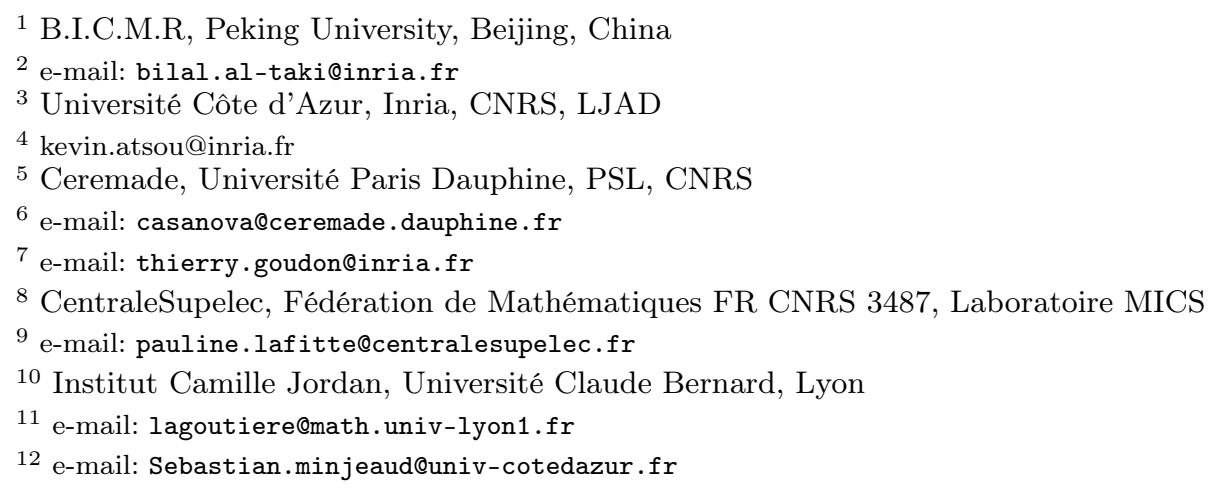

(C) EDP Sciences, SMAI 2021

This is an Open Access article distributed under the terms of the Creative Commons Attribution License (http://creativecommons.org/licenses/by/4.0), which permits unrestricted use, distribution, and reproduction in any medium, provided the original work is properly cited. 
a mere function of the density, namely we set $p(\rho)=a \rho^{\gamma}$, with $a>0$ and $\gamma>1$. Here we assume that the fluid is not subjected to external forces.

This contribution is particularly motivated by the pioneering work of D. Hoff [15]. Beyond the existence of weak solutions, 15 establishes several facts about discontinuous solutions of (1), which, at first glance, are quite surprising. Indeed, possible discontinuities of the initial velocity are instantaneously smoothed out: $u$ becomes continuous, while the density $\rho$ can present discontinuities, which are simply advected by the velocity field $u$, see $[17,26$. The velocity gradient has possible discontinuities, but cancellation occurs so that the mixed quantity $\mu \partial_{x} u-p$ is continuous. Moreover, the jump discontinuities of (1) tend to 0 exponentially fast as $t \rightarrow \infty$ : the smaller the viscosity, the faster the decay. This form is not consistent with the discontinuous solutions of the Euler equations (obtained by replacing $\mu$ by 0 in (1)). Shock solutions for the Euler system can be obtained in the vanishing viscosity regime, but discontinuities of (1) do not produce discontinuities for the inviscid system; instead the latter emerge in regular regions for the solutions of (1), see [11, 16]. For further results and references about the existence theory for the compressible Navier-Stokes system, we refer the reader to [6, 19] and the reference books [7, 22]. It is also worth mentioning the recent breakthrough [4] which deals with more intricate pressure laws and introduce new compactness arguments, and [5, 25, 27] for results on the case of density-dependent viscosities.

In terms of numerical methods, the basic idea consists in using an established method for the Euler equation, coupled to a suitable discretization of the diffusion term. In order to avoid non affordable time steps, it is quite natural to make the latter implicit. We will compare such schemes to approaches based on staggered discretizations, where the discrete densities and velocities are stored on dual locations; such methods, strongly motivated by the simulation of low-Mach flows, are discussed for instance in $8,10,13,14,20$ and in [1, 3, 12, 24], with different viewpoints on the construction of the numerical fluxes. We will also perform simulations based on Lagrangian schemes, which are able to follow more accurately the discontinuities. Nevertheless, the convergence analysis of schemes for the compressible Navier-Stokes system is not that developed: we refer the reader to [9, 18] for recent results in this direction; however, these papers investigate fully implicit schemes which could be very difficult to effectively implement (with the additional difficulties of using a Newton algorithm, which itself should be shown to converge in this context).

The paper is organized as follows. In Section 1, we present the numerical schemes that we shall use for the simulation of (1). In Section 2, we discuss a Lax-Wendroff-like statement, which provides a rigorous basis to the most naive numerical strategy. Section 3 is devoted to the discussion of the numerical results.

\section{NumericAl METHODS}

\subsection{A splitting scheme for compressible Navier-Stokes equations}

In this section, we propose a very simple scheme that consists in using a classical hyperbolic type scheme for the Euler system and adding, in a splitting procedure, the effect of the viscosity. To approximate the solution of the Euler system, we use the finite volume framework. The computational domain is the slab $(0,1)$, endowed with periodic boundary conditions for the sake of simplicity. It is discretized into $I$ cells with equal length $C_{i}=\left(x_{i-1 / 2}, x_{i+1 / 2}\right)$, and $x_{i}$ being the center of the cell. This amounts to define, for $I \in \mathbb{N}^{*}, \Delta x=1 / I$, $x_{i+1 / 2}=i \Delta x, x_{i}=(i+1 / 2) \Delta x$ for $i=0, \ldots, I$. Note that we thus have $x_{I+1 / 2}=1$, and we define also $x_{1 / 2}=0$. For the time discretization, we discuss the schemes with the generic notation $\Delta t>0$, but it has to be kept in mind that actually $\Delta t$ will have to satisfy a local in time Courant-Friedrichs-Lewy type constraint. Therefore, in fact $\Delta t$ may depend on the time step (and the local discrete time should be appropriately denoted $t^{n}=\sum_{k=1}^{n} \Delta t^{k}$ instead of $\left.n \Delta t\right)$. We denote by $X_{i}^{n}=\left(\rho_{i}^{n}, q_{i}^{n}\right)$ the approximate solution at time $t_{n}$ on the cell $C_{i}$, where $q_{i}^{n}=\rho_{i}^{n} u_{i}^{n}$ is the discrete momentum: $X_{i}^{n}$ is intended to be an approximation of $\frac{1}{\Delta x} \int_{C_{i}} X\left(t^{n}, x\right) d x$. The unknowns are updated as follows

$$
X_{i}^{n+1}=X_{i}^{n}-\frac{\Delta t}{\Delta x}\left(\mathcal{F}_{i+1 / 2}^{n}-\mathcal{F}_{i-1 / 2}^{n}\right)
$$


Here and below, we consider only first-order three points schemes where the flux $\mathcal{F}_{i+1 / 2}^{n}$ at the interface $x_{i+1 / 2}$ is defined as a function of the unknowns in the neighboring cells:

$$
\mathcal{F}_{i+1 / 2}^{n}=\mathcal{F}\left(X_{i}^{n}, X_{i+1}^{n}\right) \text {. }
$$

There are many relevant choices for the numerical fluxes. Here, we shall use the Rusanov flux, given by

$$
\mathcal{F}\left(X_{i}^{n}, X_{i+1}^{n}\right)=\frac{F\left(X_{i}^{n}\right)+F\left(X_{i+1}^{n}\right)}{2}-\max _{k \in\{1,2\}}\left(\lambda_{k}\left(X_{i}^{n}\right), \lambda_{k}\left(X_{i+1}^{n}\right)\right) \frac{X_{i+1}^{n}-X_{i}^{n}}{2} .
$$

where

$$
F(\rho, q)=\left(\begin{array}{l}
{[F(\rho, q)]_{1}} \\
{[F(\rho, q)]_{2}}
\end{array}\right)=\left(\begin{array}{c}
q \\
q^{2} / \rho+p(\rho)
\end{array}\right)
$$

and

$$
\lambda_{1}(X)=u-\sqrt{p^{\prime}(\rho)} \quad \lambda_{2}(X)=u+\sqrt{p^{\prime}(\rho)}
$$

are the eigenvalues of the Jacobian $\nabla_{\rho, q} F(\rho, q)$. We refer the reader for instance to the textbook 21 for further details about this classical scheme for conservation laws. What is crucial is the following consistency property

$$
\mathcal{F} \text { is continuous and satisfies } \mathcal{F}(X, X)=F(X) \text {. }
$$

In the convergence analysis made below, the details of the numerical fluxes are not important, except property (3). The "splitting" scheme for (1) reads

$$
\begin{gathered}
\frac{\rho_{i}^{n+1}-\rho_{i}^{n}}{\Delta t}+\frac{1}{\Delta x}\left[\left[\mathcal{F}_{i+1 / 2}^{n}\right]_{1}-\left[\mathcal{F}_{i-1 / 2}^{n}\right]_{1}\right]=0, \quad n \in \mathbb{N}, i=1, \ldots, I, \\
\frac{\rho_{i}^{n+1} u_{i}^{n+1}-\rho_{i}^{n} u_{i}^{n}}{\Delta t}+\frac{1}{\Delta x}\left[\left[\mathcal{F}_{i+1 / 2}^{n}\right]_{2}-\left[\mathcal{F}_{i-1 / 2}^{n}\right]_{2}\right]-\frac{1}{\Delta x}\left(\mathcal{D}_{i+1 / 2}^{n+1}-\mathcal{D}_{i-1 / 2}^{n+1}\right)=f_{i}^{n+1}, \\
\quad n \in \mathbb{N}, i=1, \ldots, I,
\end{gathered}
$$

where we have incorporated the diffusion flux at $x_{i+1 / 2}$, here defined by:

$$
\mathcal{D}_{i+1 / 2}^{n+1}=\mu \frac{u_{i+1}^{n+1}-u_{i}^{n+1}}{\Delta x}, \quad n \in \mathbb{N}, i=1, \ldots, I-1 .
$$

Periodic boundary conditions are taken into account by setting

$$
\mathcal{D}_{1 / 2}^{n+1}=\mu \frac{u_{1}^{n+1}-u_{I}^{n+1}}{\Delta x}=\mathcal{D}_{I+1 / 2}^{n+1}, \quad n \in \mathbb{N},
$$

with

$$
\rho_{1 / 2}^{n+1}=\frac{\rho_{1}^{n+1}+\rho_{I}^{n+1}}{2}=\rho_{I+1 / 2}^{n+1}, \quad n \in \mathbb{N} .
$$

Note that in the case where the viscosity depends on the density, (5)-(6) involves the density at time $t^{n+1}$ : since it has been already updated by (4) it can be used without any extra cost to update the velocity. 


\subsection{An explicit scheme on staggered grids}

We describe now a scheme on staggered grids: the velocity variables are stored on the cells $C_{i+1 / 2}=\left[x_{i}, x_{i+1}\right]$, while discrete densities and pressures are stored on $C_{i}=\left[x_{i-1 / 2}, x_{i+1 / 2}\right]$. The discrete mass conservation equation reads

$$
\frac{\rho_{i}^{n+1}-\rho_{i}^{n}}{\Delta t}+\frac{\mathcal{F}_{i+1 / 2}^{n}-\mathcal{F}_{i-1 / 2}^{n}}{\Delta x}=0
$$

where the mass flux $\mathcal{F}_{i+1 / 2}^{n}$ is defined from the velocity $u_{i+1 / 2}^{n}$ known at the interface $x_{i+1 / 2}$. Several definitions of the mass fluxes have been introduced, for instance by using the UpWind flux based on the material velocity [14], an idea reminiscent of the AUSM scheme [23]. Here we use instead the mass fluxes proposed in [2] which are constructed by using the characteristic speeds of the hyperbolic system. Denoting $c=\sqrt{p^{\prime}(\rho)}$ the sound speed, we set

$$
\mathcal{F}_{i+1 / 2}^{n}=\mathcal{F}^{+}\left(\rho_{i}^{n}, c_{i+1 / 2}^{n}, u_{i+1 / 2}^{n}\right)+\mathcal{F}^{-}\left(\rho_{i+1}^{n}, c_{i+1 / 2}^{n}, u_{i+1 / 2}^{n}\right)
$$

with

$$
\mathcal{F}^{+}(\rho, c, u)=\left\{\begin{array}{ll}
0 & \text { if } u \leq-c, \\
\frac{\rho}{4 c}(u+c)^{2} & \text { if }|u| \leq-c, \\
\rho u & \text { if } u \geq c,
\end{array} \quad \mathcal{F}^{-}(\rho, c, u)= \begin{cases}\rho u & \text { if } u \leq-c \\
-\frac{\rho}{4 c}(u-c)^{2} & \text { if }|u| \leq-c \\
0 & \text { if } u \geq c\end{cases}\right.
$$

and

$$
c_{i+1 / 2}^{n}=\sqrt{p^{\prime}\left(\rho_{i+1 / 2}^{n}\right)}, \quad \rho_{i+1 / 2}^{n}=\frac{\rho_{i}^{n}+\rho_{i+1}^{n}}{2} .
$$

We note that $\mathcal{F}^{ \pm} \gtrless 0$; the definition relies on the upwinding principles, according to the sign of the characteristic speeds $u \pm c$. For the momentum equation, we set

$$
\frac{\rho_{i+1 / 2}^{n+1} u_{i+1 / 2}^{n+1}-\rho_{i+1 / 2}^{n} u_{i+1 / 2}^{n}}{\Delta t}+\frac{1}{\Delta x}\left(\mathcal{G}_{i+1}^{n}-\mathcal{G}_{i}^{n}\right)+\frac{1}{\Delta x}\left(\Pi_{i+1}^{n+1 / 2}-\Pi_{i}^{n+1 / 2}\right)=\frac{\mathcal{D}_{i+1}^{n+1}-\mathcal{D}_{i}^{n+1}}{\Delta x} .
$$

In (10), a natural choice for the discretization of the pressure term would be $\Pi_{i}^{n+1 / 2}=p\left(\rho_{i}^{n}\right)$. However, it turns out that the semi-implicit formula $\Pi_{i}^{n+1 / 2}=\rho_{i}^{n} \Phi^{\prime}\left(\rho_{i}^{n+1}\right)-\Phi\left(\rho_{i}^{n}\right)$ where $\rho \Phi^{\prime}(\rho)-\Phi(\rho)=p(\rho)$, is a better choice, motivated by the consistency analysis of the scheme [2. The convection fluxes are defined by

$$
\begin{aligned}
\mathcal{G}_{i}^{n}= & \frac{u_{i-1 / 2}^{n}}{2}\left(\mathcal{F}^{+}\left(\rho_{i-1}^{n}, c_{i-1 / 2}^{n}, u_{i-1 / 2}^{n}\right)+\mathcal{F}^{+}\left(\rho_{i}^{n}, c_{i+1 / 2}^{n}, u_{i+1 / 2}^{n}\right)\right) \\
& +\frac{u_{i+1 / 2}^{n}}{2}\left(\mathcal{F}^{-}\left(\rho_{i}^{n}, c_{i-1 / 2}^{n}, u_{i-1 / 2}^{n}\right)+\mathcal{F}^{-}\left(\rho_{i+1}^{n}, c_{i+1 / 2}^{n}, u_{i+1 / 2}^{n}\right)\right)
\end{aligned}
$$

which, again, rely on upwinding principles. Finally, for the diffusion term, the definition is quite similar to (6)

$$
\mathcal{D}_{i}^{n+1}=\mu \frac{u_{i+1 / 2}^{n+1}-u_{i-1 / 2}^{n+1}}{\Delta x} .
$$

Relations (9) and (10) hold for $i=1, \ldots, I$, imposing furthermore $\rho_{I+1}^{n}=\rho_{1}^{n}, u_{1 / 2}^{n}=u_{I+1 / 2}^{n}, \mathcal{D}_{I+1}^{n+1}=$ $\mu \frac{u_{3 / 2}^{n+1}-u_{I+1 / 2}^{n+1}}{\Delta x}=\mathcal{D}_{1}^{n+1}$.

In the following, this scheme will be referred to as Eulerian staggered scheme 1. 


\subsection{A staggered pseudo-Lagrangian scheme}

In this section we propose a pseudo-Lagrangian scheme. We use the term "pseudo-Lagangrian" because although it approximates the solution in the Euler variables, it strongly uses the conservation of the quantities in material volumes. In this scheme, the density unknowns at time $t^{n}, \rho_{j}^{n}$, are inside the cells $\left(x_{j-1 / 2}^{n}, x_{j+1 / 2}^{n}\right)$, which are time-dependent, and the velocity unknowns $u_{j+1 / 2}^{n}$ are associated with the boundaries of the cells, the $x_{j+1 / 2}^{n}$ 's. The latter are advected at velocity $u_{j+1 / 2}^{n}$. The discretization of the mass equation corresponds to the discretization of the relation

$$
\frac{d}{d t} \int_{x(t)}^{y(t)} \rho(t, x) d x=0
$$

with $x(t)$ and $y(t)$ moving at the fluid velocity. It expresses that the mass in every cell is constant in time. Namely, we obtain

$$
\left\{\begin{array}{l}
x_{j+1 / 2}^{n+1}=x_{j+1 / 2}^{n}+\Delta t u_{j+1 / 2}^{n}, \quad n \in \mathbb{N}, j=1, \ldots, J \\
x_{1 / 2}^{n+1}=x_{J+1 / 2}^{n+1}-1 \quad n=-1, \ldots,+\infty \\
\Delta x_{j}^{n+1}=x_{j+1 / 2}^{n+1}-x_{j-1 / 2}^{n+1}, \quad n=-1, \ldots,+\infty, j=1, \ldots, J \\
\rho_{j}^{n+1}=\rho_{j}^{n} \Delta x_{j}^{n} / \Delta x_{j}^{n+1}, \quad n \in \mathbb{N}, j \in 1, \ldots, J .
\end{array}\right.
$$

The second equation in (11) accounts for the periodicity of the problem.

We turn to the momentum equation $\partial_{t}(\rho u)+\partial_{x}\left(\rho u^{2}\right)=\partial_{x}\left(\mu \partial_{x} u-p\right)$, which is interpreted as

$$
\frac{d}{d t} \int_{x(t)}^{y(t)} \rho(t, x) u(t, x) d x=\mu \partial_{x} u(t, y(t))-p(t, y(t))-\left(\mu \partial_{x} u(t, x(t))-p(t, x(t))\right) .
$$

It is approximated by

$$
\left\{\begin{array}{l}
\rho_{j+1 / 2}^{n+1} u_{j+1 / 2}^{n+1} \Delta x_{j+1 / 2}^{n+1}=\rho_{j+1 / 2}^{n} u_{j+1 / 2}^{n} \Delta x_{j+1 / 2}^{n} \\
\quad-\left(p_{j+1}^{n+1}-p_{j}^{n+1}\right)+\mu\left(\frac{u_{j+3 / 2}^{n+1}-u_{j+1 / 2}^{n+1}}{\Delta x_{j+1}^{n+1}}-\frac{u_{j+1 / 2}^{n+1}-u_{j-1 / 2}^{n+1}}{\Delta x_{j}^{n+1}}\right), \quad n \in \mathbb{N}, j=1, \ldots J \\
u_{1 / 2}^{n+1}=u_{J+1 / 2}^{n+1}, \quad n \in \mathbb{N}, \\
u_{J+3 / 2}^{n+1}=u_{3 / 2}^{n+1}, \quad n \in \mathbb{N} \\
p_{J+1}^{n+1}=p_{1}^{n+1}, \quad n \in \mathbb{N}, \\
\Delta x_{J+1}^{n+1}=\Delta x_{1}^{n+1}, \quad n \in \mathbb{N},
\end{array}\right.
$$

where the last four equations stand for the periodicity. In these formulae, we use the quantities $\rho_{j+1 / 2}^{n+1}, \Delta x_{j+1 / 2}^{n+1}$ and $p_{j}^{n+1}$ that were not defined previously: we choose for them the following rather natural definition:

$$
\left\{\begin{array}{l}
\Delta x_{j+1 / 2}^{n+1}=\left(\Delta x_{j}^{n+1}+\Delta x_{j+1}^{n+1}\right) / 2, \quad n=-1, \ldots,+\infty, j=1, \ldots, J \\
\rho_{j+1 / 2}^{n+1} \Delta x_{j+1 / 2}^{n+1}=\left(\rho_{j}^{n+1} \Delta x_{j}^{n+1}+\rho_{j+1}^{n+1} \Delta x_{j+1}^{n+1}\right) / 2, \quad n=-1, \ldots,+\infty, j=1, \ldots, J \\
p_{j}^{n+1}=p\left(\rho_{j}^{n+1}\right), \quad n=-1, \ldots,+\infty, j=1, \ldots, J
\end{array}\right.
$$

Complemented with initial conditions $\rho_{j}^{0}, u_{j+1 / 2}^{0}, x_{j+1 / 2}^{0}$, the scheme is defined. Note that we have chosen an implicit approximation for the diffusion, in order to avoid any parabolic stability condition (and indeed we observe the stability of the algorithm).

\subsection{A last staggered scheme}

In this section we propose another staggered scheme, that can be viewed as a Eulerian version of the preceding pseudo-Lagrangian one. Here the cells are fixed and, starting from the pseudo-Lagrangian scheme, we have to 
take the transport into account. With the same notations, but now the length $\Delta x$ of the cells is constant, the mass conservation is replaced with

$$
\rho_{j}^{n+1}=\rho_{j}^{n}-\frac{\Delta t}{\Delta x}\left(\rho_{j+1 / 2, U}^{n} u_{j+1 / 2}^{n}-\rho_{j-1 / 2, U}^{n} u_{j-1 / 2}^{n}\right), \quad n \in \mathbb{N}, j \in 1, \ldots, J,
$$

where $\rho_{j+1 / 2, U}^{n}$ is upwinded, that is to say that it is $\rho_{j}^{n}$ if $u_{j+1 / 2}^{n} \geq 0$ and $\rho_{j+1 / 2}^{n}$ if $u_{j+1 / 2}^{n}<0$.

The momentum equation is approximated by

$$
\left\{\begin{array}{l}
\rho_{j+1 / 2}^{n+1} u_{j+1 / 2}^{n+1}=\rho_{j+1 / 2}^{n} u_{j+1 / 2}^{n}-\frac{\Delta t}{2 \Delta x}\left(\rho_{j+3 / 2}^{n+1}\left|u_{j+3 / 2}^{n}\right|^{2}-\rho_{j-1 / 2}^{n+1}\left|u_{j-1 / 2}^{n}\right|^{2}\right) \\
\quad-\frac{\Delta t}{\Delta x}\left(p_{j+1}^{n+1}-p_{j}^{n+1}\right)+\mu \frac{\Delta t}{\Delta x^{2}}\left(u_{j+3 / 2}^{n+1}-2 u_{j+1 / 2}^{n+1}+u_{j-1 / 2}^{n+1}\right), \quad n \in \mathbb{N}, j=1, \ldots J, \\
u_{1 / 2}^{n+1}=u_{J+1 / 2}^{n+1}, \quad n \in \mathbb{N}, \\
u_{J+3 / 2}^{n+1}=u_{3 / 2}^{n+1}, \quad n \in \mathbb{N}, \\
p_{J+1}^{n+1}=p_{1}^{n+1}, \quad n \in \mathbb{N}, \\
\Delta x_{J+1}^{n+1}=\Delta x_{1}^{n+1}, \quad n \in \mathbb{N},
\end{array}\right.
$$

where the last four equations stand for the periodicity. In these formulae, we use the quantities $\rho_{j+1 / 2}^{n+1}$ and $p_{j}^{n+1}$ that were not defined previously: we choose for them the following rather natural definition:

$$
\left\{\begin{array}{l}
\rho_{j+1 / 2}^{n+1}=\left(\rho_{j}^{n+1}+\rho_{j+1}^{n+1}\right) / 2, \quad n=-1, \ldots,+\infty, j=1, \ldots, J \\
p_{j}^{n+1}=p\left(\rho_{j}^{n+1}\right), \quad n=-1, \ldots,+\infty, j=1, \ldots, J
\end{array}\right.
$$

Complemented with initial conditions $\rho_{j}^{0}, u_{j+1 / 2}^{0}, x_{j+1 / 2}^{0}$, the scheme is defined. Note that we have chosen an implicit approximation for the diffusion, in order to avoid any parabolic stability condition (and indeed we observe the stability of the algorithm). The convection term in the momentum equation is centered, but for fixed (not too small) viscosity coefficient, this does not make instabilities appear.

In the following, this scheme will be referred to as Eulerian staggered scheme 2.

\section{Convergence Analysis à la LAX-Wendroff}

In this section, we investigate the convergence of the Rusanov scheme. For the sake of simplicity, we restrict the analysis to the case of uniform meshes: all cells have the same length. We consider a sequence of meshes, parametrized by $k \in \mathbb{N}$, such that $\Delta x_{k}$ and $\Delta t_{k}$ both tend to 0 , with $\frac{\Delta x_{k}}{\Delta t_{k}}=\lambda$ for some $\lambda>0$ to ensure some stability property, for instance the positivity of the density.

The discrete initial data are given by

$$
\rho_{j}^{0}=\frac{1}{\Delta x_{k}} \int_{x_{j-1 / 2}}^{x_{j+1 / 2}} \rho^{0}(x) d x, \quad u_{j}^{0}=\frac{1}{\Delta x_{k}} \int_{x_{j-1 / 2}}^{x_{j+1 / 2}} u^{0}(x) d x \text { for all } j \in\{1, \ldots, I\} .
$$

The discrete approximation of $\rho$ and $u$ are denoted by

$$
\begin{aligned}
\rho_{k}(t, x) & =\sum_{n=0}^{N-1} \sum_{j=1}^{I} \rho_{j}^{n} \mathbb{1}_{\left[t^{n}, t^{n+1}\right)}(t) \mathbb{1}_{\left[x_{j-1 / 2}, x_{j+1 / 2}\right)}(x), \\
u_{k}(t, x) & =\sum_{n=0}^{N-1} \sum_{j=1}^{I} u_{j}^{n} \mathbb{1}_{\left[t^{n}, t^{n+1}\right)}(t) \mathbb{1}_{\left[x_{j-1 / 2}, x_{j+1 / 2}\right)}(x) .
\end{aligned}
$$

We bear in mind that $\rho_{j}^{n}$ and $u_{j}^{n}$ also depend on $k$ but this dependency is omitted to simplify the notation. The following statement is in the same spirit as the standard Lax-Wendroff theorem for conservation laws, see [21, Section 12.10]. 
Theorem 2.1. Suppose that

- there exists a constant $C_{\infty}>0$ such that

$$
\sup _{k}\left(\left\|u_{k}\right\|_{L^{\infty}((0, T) \times(0,1))}+\left\|\rho_{k}\right\|_{L^{\infty}((0, T) \times(0,1))}\right) \leq C_{\infty} .
$$

- there exists $(\rho, u) \in L^{\infty}((0, T) \times(0,1))^{2}$ such that $\left(\rho_{k}, u_{k}\right)$ converges to $(\rho, u)$ in $L^{r}((0, T) \times(0,1))^{2}$ for any $1 \leq r<+\infty$.

Then the pair $(u, \rho)$ is a weak solution of (1).

Remark 2.2. The statement remains true when $\mu(\rho)=C \rho$ at the price of assuming

$$
u \in L^{r}\left(0, T ; W^{1, r}(0,1)\right)
$$

and

$$
\frac{u_{k}\left(t, x+\Delta x_{k}\right)-u_{k}(t, x)}{\Delta x_{k}} \rightarrow \nabla u(t, x) \text { weakly in } L^{r}((0, T) \times(0,1)) .
$$

It equally applies for a continuous function $\mu$, with the additional assumptions that the convergence of $\left(\rho_{k}, u_{k}\right)$ to $(\rho, u)$ holds almost everywhere.

Proof. Consider a test function $\zeta=(\varphi, \chi) \in \mathcal{C}_{0}^{\infty}\left([0, T) \times(0,1) ; \mathbb{R}^{2}\right)$. We assume that $\Delta t_{k}$ and $\Delta x_{k}$ are small enough so that $\operatorname{supp}(\zeta) \subset\left[0, T-\Delta t_{k}\right) \times\left(2 \Delta x_{k}, 1-2 \Delta x_{k}\right)$. For $n \in\{0, \ldots, N-1\}$ and $j \in\{1, \ldots, I\}$, let us denote $\zeta_{j}^{n}=\zeta\left(t^{n}, x_{j}\right)$, and, for all $t \in(0, T), x \in(0,1)$,

$$
\zeta_{\Delta t_{k}, \Delta x_{k}}(t, x)=\sum_{n=0}^{N-1} \sum_{j=1}^{I} \zeta_{j}^{n} \mathbb{1}_{\left[t^{n}, t^{n+1}\right)}(t) \mathbb{1}_{\left[x_{j-1 / 2}, x_{j+1 / 2}\right)}(x), \quad \zeta_{k}=\zeta_{\Delta t_{k}, \Delta x_{k}} .
$$

We multiply the discrete mass conservation equation by $\Delta x_{k} \Delta t_{k} \varphi_{j}^{n}$, and we sum over $j$ and $n$. We obtain

$$
\Delta x_{k} \sum_{n=0}^{N-1} \sum_{j=1}^{I}\left(\rho_{j}^{n+1}-\rho_{j}^{n}\right) \varphi_{j}^{n}+\Delta t_{k} \sum_{n=0}^{N-1} \sum_{j=1}^{I}\left[\left[\mathcal{F}_{j+1 / 2}^{n}\right]_{1}-\left[\mathcal{F}_{j-1 / 2}^{n}\right]_{1}\right] \varphi_{j}^{n}=0 .
$$

The first term on the left hand side recasts as

$$
\Delta x_{k} \sum_{n=0}^{N-2} \sum_{j=1}^{I} \rho_{j}^{n+1}\left(\varphi_{j}^{n}-\varphi_{j}^{n+1}\right)+\Delta x_{k} \underbrace{\sum_{j=1}^{I} \rho_{j}^{N} \varphi_{j}^{N}}_{=0}-\Delta x_{k} \sum_{j=1}^{I} \rho_{j}^{0} \varphi_{j}^{0}
$$

while the second term becomes

$$
\Delta t_{k} \sum_{n=0}^{N-1} \sum_{j=1}^{I-1}\left[\mathcal{F}_{j+1 / 2}^{n}\right]_{1}\left(\varphi_{j}^{n}-\varphi_{j+1}^{n}\right)+\underbrace{\Delta t_{k} \sum_{n=0}^{N-1}\left[\mathcal{F}_{I+1 / 2}^{n}\right]_{1} \varphi_{I}^{n}-\Delta t_{k} \sum_{n=0}^{N-1}\left[\mathcal{F}_{1 / 2}^{n}\right]_{1} \varphi_{1 / 2}^{n}}_{=0} .
$$

Hence we get

$$
\Delta x_{k} \sum_{n=0}^{N-2} \sum_{j=1}^{I} \rho_{j}^{n+1}\left(\varphi_{j}^{n}-\varphi_{j}^{n+1}\right)+\Delta t_{k} \sum_{n=0}^{N-1} \sum_{j=1}^{I-1}\left[\mathcal{F}_{j+1 / 2}^{n}\right]_{1}\left(\varphi_{j}^{n}-\varphi_{j+1}^{n}\right)=\Delta x_{k} \sum_{j=1}^{I} \rho_{j}^{0} \varphi_{j}^{0} .
$$


Using (3), the estimates and convergence assumed for $\left(\rho_{k}, u_{k}\right)$, we obtain as $k \rightarrow \infty$

$$
\begin{aligned}
& \Delta x_{k} \sum_{n=0}^{N-2} \sum_{j=1}^{I} \rho_{j}^{n+1}\left(\varphi_{j}^{n}-\varphi_{j}^{n+1}\right) \rightarrow \int_{0}^{T} \int_{0}^{1} \rho \partial_{t} \varphi d x d t, \\
& \Delta t_{k} \sum_{n=0}^{N-1} \sum_{j=1}^{J-1}\left[F_{j+1 / 2}^{n}\right]_{1}\left(\varphi_{j}^{n}-\varphi_{j+1}^{n}\right) \rightarrow \int_{0}^{T} \int_{0}^{1} \rho u \partial_{x} \varphi d x d t, \\
& \Delta x_{k} \sum_{j=1}^{I} \rho_{j}^{0} \varphi_{j}^{0} \rightarrow \int_{0}^{1} \rho^{0}(x) \varphi(0, x) d x .
\end{aligned}
$$

We turn to the momentum equation (5). Proceeding similarly, we obtain

$$
\begin{aligned}
& \Delta x_{k} \sum_{n=0}^{N-1} \sum_{j=1}^{I}\left(\rho_{j}^{n+1} u_{j}^{n+1}-\rho_{j}^{n} u_{j}^{n}\right) \chi_{j}^{n}+\Delta t_{k} \sum_{n=0}^{N-1} \sum_{j=1}^{I}\left[\left[\mathcal{F}_{j+1 / 2}^{n}\right]_{2}-\left[\mathcal{F}_{j-1 / 2}^{n}\right]_{2}\right] \chi_{j}^{n} \\
& -\Delta t_{k} \sum_{n=0}^{N-1} \sum_{j=1}^{I}\left[\mu\left(\rho_{j+1 / 2}^{n+1}\right) \frac{u_{j+1}^{n+1}-u_{j}^{n+1}}{\Delta x_{k}}-\mu\left(\rho_{j-1 / 2}^{n+1}\right) \frac{u_{j}^{n+1}-u_{j-1}^{n+1}}{\Delta x_{k}}\right] \chi_{j}^{n}=0 .
\end{aligned}
$$

The first two sums can be written as

$$
\Delta x_{k} \sum_{n=0}^{N-2} \sum_{j=1}^{I-1} \rho_{j}^{n+1} u_{j}^{n+1}\left(\chi_{j}^{n}-\chi_{j}^{n+1}\right)+\Delta t_{k} \sum_{n=0}^{N-1} \sum_{j=1}^{I-1}\left[\mathcal{F}_{j+1}^{n}\right]_{2}\left(\chi_{j}^{n}-\chi_{j+1}^{n}\right),
$$

which can be treated as for the mass conservation equation, by using that the product $\rho_{k} u_{k}$ still converges in $L^{r}((0, T) \times(0,1))$ for $1 \leq r<+\infty$. Let us study the viscous term

$$
\Delta t_{k} \sum_{n=0}^{N-1} \sum_{j=1}^{I}\left[\mu\left(\rho_{j+1 / 2}^{n+1}\right) \frac{u_{j+1}^{n+1}-u_{j}^{n+1}}{\Delta x_{k}}-\mu\left(\rho_{j-1 / 2}^{n+1}\right) \frac{u_{j}^{n+1}-u_{j-1}^{n+1}}{\Delta x_{k}}\right] \chi_{j}^{n} .
$$

The case where the viscosity $\mu$ is constant can be handled by using two summations by parts, which allow us to write 19 as

$$
\Delta t_{k} \sum_{n=0}^{N-1} \sum_{j=2}^{I-1} u_{j}^{n+1} \frac{\chi_{j+1}^{n}-2 \chi_{j}^{n}+\chi_{j-1}^{n}}{\Delta x_{k}}
$$

This can be cast as

$$
\int_{0}^{T-\Delta t_{k}} \int_{2 \Delta x_{k}}^{1-2 \Delta x_{k}} u_{k}\left(t+\Delta t_{k}, x\right) \frac{\chi_{k}\left(t, x+\Delta x_{k}\right)-2 \chi_{k}(t, x)+\chi_{k}\left(t, x-\Delta x_{k}\right)}{\Delta x_{k}^{2}} d x d t,
$$

and using the uniform convergence of $\chi_{k}$ and its derivatives, together with the convergence in $L^{r}$ of $u_{k}$, we are able to pass to the limit: as $k \rightarrow \infty$, we obtain

$$
\int_{0}^{T} \int_{0}^{1} u(t, x) \partial_{x x}^{2} \chi(t, x) d x d t
$$

which completes the proof in the case of constant viscosity. 
When $\mu$ depends on the density, we cannot proceed this way, and we need further assumptions. We start by writing 19 as

$$
\begin{gathered}
\Delta t_{k} \sum_{n=0}^{N-1} \sum_{j=1}^{I}\left[\mu\left(\rho_{j+1 / 2}^{n+1}\right) \frac{u_{j+1}^{n+1}-u_{j}^{n+1}}{\Delta x_{k}}-\mu\left(\rho_{j-1 / 2}^{n+1}\right) \frac{u_{j}^{n+1}-u_{j-1}^{n+1}}{\Delta x_{k}}\right] \chi_{j}^{n} \\
=\int_{\Delta t_{k}}^{T} \int_{0}^{1} \mu\left(\frac{\rho_{k}\left(t, x_{k}+\Delta x_{k}\right)+\rho_{k}(t, x)}{2}\right) \frac{u_{k}\left(t, x+\Delta x_{k}\right)-u_{k}(t, x)}{\Delta x_{k}} \\
\times \frac{\chi_{k}\left(t-\Delta t_{k}, x\right)-\chi_{k}\left(t-\Delta t, x+\Delta x_{k}\right)}{\Delta x_{k}} d x d t .
\end{gathered}
$$

To pass to the limit in the previous term we use a weak-strong convergence argument observing that

$$
\begin{aligned}
& \mu\left(\frac{\rho_{k}\left(t, x_{k}+\Delta x_{k}\right)+\rho_{k}(t, x)}{2}\right) \frac{\chi_{k}\left(t-\Delta t_{k}, x\right)-\chi_{k}\left(t-\Delta t_{k}, x+\Delta x_{k}\right)}{\Delta x_{k}} \\
& \underset{k \rightarrow \infty}{\longrightarrow} \mu(\rho(t, x)) \partial_{x} \chi(t, x) \text { in } L^{r^{\prime}}((0, T) \times(0,1)) .
\end{aligned}
$$

This combines to the assumption that $\nabla u_{k}$ converges weakly in $L^{r}((0, T) \times(0,1))$ so that 20 tends to

$$
\int_{0}^{T} \int_{0}^{1} \mu(\rho(t, x)) \partial_{x} u(t, x) \partial_{x} \chi(t, x) d x d t
$$

which concludes the proof.

\section{NumERICAL RESULTS}

Here, we present some numerical results that illustrate the behavior of the proposed schemes.

\subsection{Comparisons}

In this subsection, we compare the results obtained with the 4 different schemes that are dealt with in the paper. The results here are obtained at time $t=0.1$ with $\mu=0.1$ for the initial condition $u^{0}(x)=0$ and $\rho^{0}(x)=0.125+1.875 \chi_{[1 / 4,3 / 4]}(x)$. 

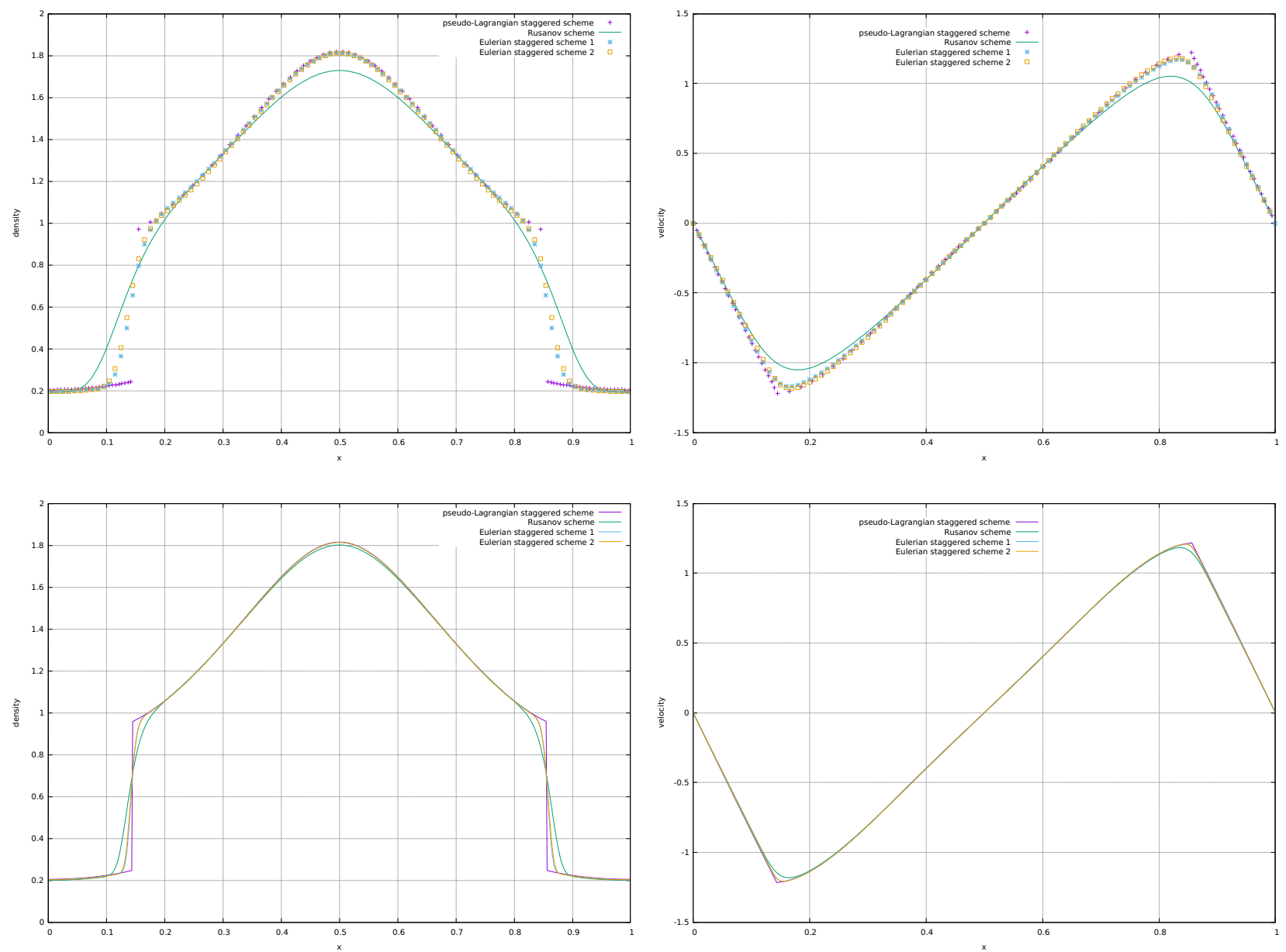

Figure 1. Density and velocity solutions at time 0.1, with 100 cells (top) and 800 cells (bottom).

We see that the four schemes seem to converge to the same solution, and that the pseudo-Lagrange scheme is less diffusive (the scheme based on the Rusanov flux is the most diffusive). In particular, the pseudo-Lagrange scheme is able to maintain some discontinuities in the density and in the velocity derivative.

For the same test-case, we now more precisely compare these four schemes, in the velocity variable. In Table 1 we compare, for different numbers of cells, the non-diffusive pseudo-Lagrangian scheme from Section 1.3 and the Eulerian Rusanov scheme from Section 1.1. The difference between these schemes seems to be of order $1 / 2$, both in the $L^{1}$ and in the $L^{\infty}$ norms. Note that the schemes are subject to some hyperbolic-typed Courant-Friedrichs-Lewy conditions, so that the time step is of the same order as the space step. Note also that to compare the solutions, as they are not obtained on the same meshes (the pseudo-Lagrange scheme is running on a moving mesh), we interpolate the difference of the two solutions on a mesh that is the intersection of the two and consider both solutions as constant-by-cell. In Table 2 we compare the pseudo-Lagrange and the Eulerian staggered scheme 1 presented in Section 1.2 . In Table 3 we compare the pseudo-Lagrange and the Eulerian staggered scheme 2 form Section 1.4. We observe the same order of convergence. 


\begin{tabular}{|c|c|c|}
\hline Number of cells $J$ & Difference in $L^{1}$ norm & Difference in $L^{\infty}$ norm \\
\hline 100 & 0.734125 & 0.247230 \\
\hline 200 & 0.038315 & 0.171206 \\
\hline 400 & 0.019801 & 0.110994 \\
\hline 800 & 0.010399 & 0.077385 \\
\hline 1600 & 0.005710 & 0.052437 \\
\hline 3200 & 0.003376 & 0.037031 \\
\hline 6400 & 0.002201 & 0.251310 \\
\hline 12800 & 0.001535 & 0.017738 \\
\hline 25600 & 0.001090 & 0.012551 \\
\hline
\end{tabular}

TABLE 1. Difference between the pseudo-Lagrange scheme and the Rusanov scheme.

\begin{tabular}{|c|c|c|}
\hline Number of cells $J$ & Difference in $L^{1}$ norm & Difference in $L^{\infty}$ norm \\
\hline 100 & 0.031244 & 0.116505 \\
\hline 200 & 0.015694 & 0.076648 \\
\hline 400 & 0.007999 & 0.048226 \\
\hline 800 & 0.004164 & 0.033637 \\
\hline 1600 & 0.002299 & 0.022405 \\
\hline 3200 & 0.001393 & 0.015951 \\
\hline 6400 & 0.000913 & 0.010339 \\
\hline 12800 & 0.000629 & 0.007328 \\
\hline 25600 & 0.000445 & 0.005201 \\
\hline
\end{tabular}

TABLE 2. Difference between the Pseudo-Lagrange scheme and the Euler staggered scheme 1.

\begin{tabular}{|c|c|c|}
\hline Number of cells $J$ & Difference in $L^{1}$ norm & Difference in $L^{\infty}$ norm \\
\hline 100 & 0.022931 & 0.109549 \\
\hline 200 & 0.012467 & 0.062358 \\
\hline 400 & 0.007078 & 0.048725 \\
\hline 800 & 0.004213 & 0.030653 \\
\hline 1600 & 0.002638 & 0.023727 \\
\hline 3200 & 0.001723 & 0.016027 \\
\hline 6400 & 0.001159 & 0.011299 \\
\hline 12800 & 0.000793 & 0.008065 \\
\hline 25600 & 0.000549 & 0.005608 \\
\hline
\end{tabular}

TABle 3. Difference between the Pseudo-Lagrange scheme and the Euler staggered scheme 2.

\subsection{Behavior of the pseudo-Lagrange scheme on Hoff-type discontinuous solutions}

In this section, we would like to illustrate a very interesting property of discontinuous solutions à la Hoff. These solutions, among which one finds the one illustrated in the previous section, have been studied by Hoff (see [15]). They consist in solutions with discontinuities in density, and thus in pressure and in the derivative of the velocity (because the effective flux $p-\mu \partial_{x} u$ is continuous in space for almost every time). In [15] it is shown that the amplitude of the jump in $\log (\rho)$ decreases exponentially in time, with a rate that is at least some constant over the viscosity coefficient $\mu$. We do not precise the result here because the constant depends 
on the maximum value of the density in the flow, and because actually the context of [15] is a bit different: it considers the Navier-Stokes system in Lagrange variables with a space variable ranging over $\mathbb{R}$, with a single discontinuity, while here we consider the Navier-Stokes system on the torus. We propose to analyse numerically the behavior of the jump of $\log (\rho)$. This is made possible thanks to the use of a pseudo-Lagrange scheme that does not smooth the discontinuity in $\rho$ artificially. With the same initial condition as in the previous section, Figure 2 presents the amplitude of the jump in $\log (\rho)$ with respect to time, with 500 cells in space. We also present its best approximation as $\exp (a+b t)$ by computing $a$ and $b$ by the least square method. Note that it is not relevant to do this on a too large time interval (with a given cell size), because the amplitude of the jump decays exponentially fast and it rapidly becomes of the same order as the cell size so that it cannot be distinguished from the natural steps of the discrete solution in regular regions.

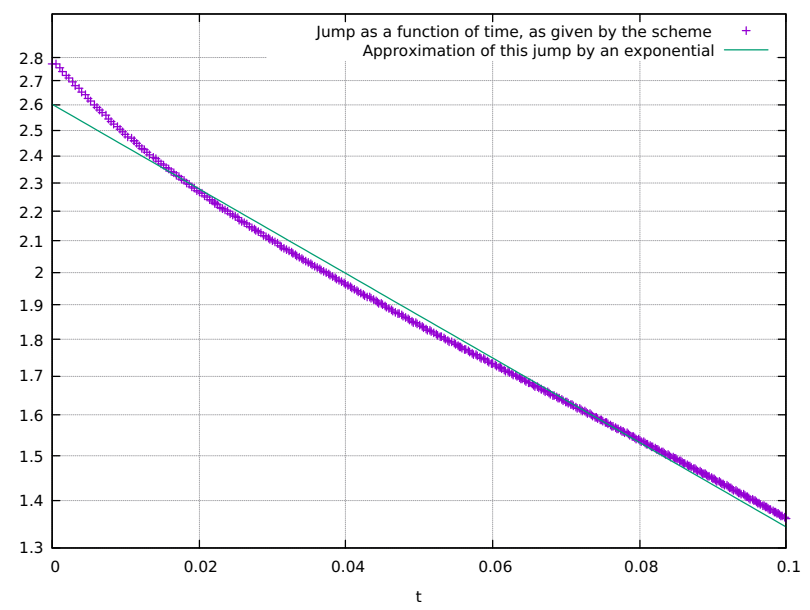

Figure 2. Jump of the quantity $\log (\rho)$ as a function of time.

Now we want to evaluate the rate at which the jump decreases as a function of the viscosity coefficient. Table 4 provides the coefficient $b$ computed by the least square method, for various values of $\mu$.

\begin{tabular}{|c|c|}
\hline Viscosity coefficient $\mu$ & Rate \\
\hline 0.1 & -6.62 \\
\hline 0.05 & -11.05 \\
\hline 0.025 & -22.17 \\
\hline 0.0125 & -54.04 \\
\hline 0.0675 & -105.14 \\
\hline
\end{tabular}

TABLE 4. Rate for the (decreasing) amplitude of the jump of the logarithm of $\rho$.

We note in Table 4 that the jump $[\log (\rho)]$ (with usual notations for the jump of a quantity) seems to behave as $\exp (-C t / \mu)$, which is exactly the majoration proved in [15]. Note that this is a very interesting phenomenon: as $\mu$ tends to 0 , this kind of discontinuities do not persist at all (they disappear at time $t=0^{+}$), what is a paradox, as for compressible inviscid gases discontinuities are expected (and they are called shocks). This result by Hoff thus proves that the shocks in inviscid gases are limits of smooth parts in the solutions of viscous gases, in which the amplitude of discontinuities tends to 0 . 


\section{REFERENCES}

[1] F. Berthelin, T. Goudon, and S. Minjeaud. Consistency analysis of a 1D finite volume scheme for barotropic Euler models. In J. Fuhrmann, M. Ohlberger, and C. Rohde, editors, Finite Volumes for Complex Applications VII; Methods, Theoretical Aspects, and Elliptic, Parabolic and Hyperbolic Problems, Berlin, volume 77 and 78 of Springer Proceedings in Mathematics ES Statistics, pages 97-106. Springer, 2014.

[2] F. Berthelin, T. Goudon, and S. Minjeaud. Kinetic schemes on staggered grids for barotropic Euler models: entropy-stability analysis. Math. Comput., 84:2221-2262, 2015.

[3] F. Berthelin, T. Goudon, and S. Minjeaud. Multifluid flows: a kinetic approach. J. Sci. Comput., 66(2):792-824, 2016.

[4] D. Bresch and P. E. Jabin. Global existence of weak solutions for compressible Navier-Stokes equations: thermodynamically unstable pressure and anisotropic viscous stress tensor. Ann. Math., 188(2):577-684, 2018.

[5] D. Bresch, A. Vasseur, and Ch. Yu. Global existence of entropy-weak solutions to the compressible Navier-Stokes equations with non-linear density dependent viscosities. Technical report, Univ. Texas Austin, 2019.

[6] G.-Q. Chen, D. Hoff, and K. Trivisa. Global solutions of the compressible Navier-Stokes equations with large discontinuous initial data. Comm. Partial Differential Equations, 25:2233-2257, 2000.

[7] E. Feireisl. Dynamics of viscous compressible fluids, volume 26 of Oxford Lecture Series in Mathematics and its Applications. Oxford University Press, Oxford, 2004.

[8] T. Gallouët, R. Herbin, and J.-C. Latché. Kinetic energy control in explicit finite volume discretizations of the incompressible and compressible Navier-Stokes equations. Int. J. Finite Vol., 7(2):6, 2010.

[9] T. Gallouet, R. Herbin, D. Maltese, and A. Novotny. Error estimates for a numerical approximation to the compressible barotropic Navier-Stokes equations. IMA J. Numer. Anal., 36(2):543-592, 2016.

[10] L. Gastaldo, R. Herbin, W. Kheriji, C. Lapuerta, and J.-C. Latché. Staggered discretizations, pressure correction schemes and all speed barotropic flows. In Finite Volumes for Complex Applications VI, Problems and Perspectives, Prague, Czech Republic, volume 4, pages 839-855, 2011.

[11] D. Gilbarg. The existence and limit behavior of the one-dimensional shock layer. Amer. J. Math., 73:256-274, 1951.

[12] T. Goudon, J. Llobell, and S. Minjeaud. An explicit MUSCL scheme on staggered grids with kinetic-like fluxes for the barotropic and full Euler system. Comm. Comput. Phys., 2020. To appear.

[13] R. Herbin, W. Kheriji, and J.-C. Latché. Staggered schemes for all speed flows. ESAIM:Proc, 35:122-150, 2012. Actes du Congrès National de Mathématiques Appliquées et Industrielles.

[14] R. Herbin, J.-C. Latché, and T. T. Nguyen. Explicit staggered schemes for the compressible Euler equations. In Applied mathematics in Savoie-AMIS 2012: Multiphase flow in industrial and environmental engineering, volume 40 of ESAIM Proc., pages 83-102. EDP Sci., Les Ulis, 2013.

[15] D. Hoff. Global existence for 1D, compressible, isentropic Navier-Stokes equations with large initial data. Trans. Amer. Math. Soc., 303(1):169-181, 1987.

[16] D. Hoff and T.-P. Liu. The inviscid limit for the Navier-Stokes equations of compressible, isentropic flow with shock data. Indiana Univ. Math. J., 38(4):861-915, 1989.

[17] D. Hoff and J. Smoller. Solutions in the large for certain nonlinear parabolic systems. Ann. Inst. Henri Poincaré, Anal. Non lin., 2:213-235, 1985.

[18] T. K. Karper. Convergent finite differences for $1 \mathrm{D}$ viscous isentropic flow in Eulerian coordinates. Disc. Cont. Dyn. Syst.- S, 7(5):993-1023, 2014.

[19] A. V. Kazhikhov and V. V. Shelukhin. Unique global solution with respect to time of initial-boundary value problems for one-dimensional equations of a viscous gas. Prikl. Mat. Meh., 41:282-291, 1977.

[20] W. Kheriji, R. Herbin, and J.-C. Latché. Pressure correction staggered schemes for barotropic one-phase and two-phase flows. Comput. ES Fluids, 88:524 - 542, 2013.

[21] R. J. Leveque. Finite Volume Methods for Hyperbolic Problems. Cambridge Texts in Appl. Math. Cambridge Univ. Press, 2004 .

[22] P.-L Lions. Mathematical topics in fluid mechanics. Vol. 2: Compressible models, volume 10 of Oxford Lecture Series in Mathematics and its Applications. The Clarendon Press, Oxford University Press, New York, 1998. Compressible models, Oxford Science Publications.

[23] M.-S. Liou and C. J. Steffen Jr. A new flux splitting scheme. J. Comput. Phys., 107:23-39, 1993.

[24] J. Llobell. Schémas Volumes Finis à mailles décalées pour la dynamique des gaz. PhD thesis, Université Côte d'Azur, 2018.

[25] A. Mellet and A. Vasseur. On the barotropic compressible Navier-Stokes equations. Comm. PDE, 32(3):431-452, 2007.

[26] D. Serre. Variations de grande amplitude pour la densité d'un fluide visqueux compressible. Phys. D, 48(1):113-128, 1991.

[27] A. Vasseur and Ch. Yu. Existence of global weak solutions for 3D degenerate compressible Navier-Stokes equations. Invent. Math., 206(3):935-974, 2016. 